\title{
Small-scale properties of the KPZ equation and dynamical symmetry breaking
}

\author{
David Hochberg ${ }^{+, *}$, Carmen Molina-París ${ }^{++, *}$, Juan Pérez-Mercader ${ }^{+, *}$, and Matt Visser ${ }^{+++}$ \\ ${ }^{+}$Laboratorio de Astrofísica Espacial y Física Fundamental, Apartado 50727, 28080 Madrid, Spain \\ ${ }^{++}$Theoretical Division, Los Alamos National Laboratory, Los Alamos, New Mexico 87545, USA \\ ${ }^{+++}$Physics Department, Washington University, Saint Louis, Missouri 63130-4899, USA \\ ${ }^{*}$ Centro de Astrobiología, INTA-CSIC, Ctra. Ajalvir, Km. 4, 28850 Torrejón, Madrid, Spain
}

(Revised April 2000; LATEX-ed January 2, 2018)

A functional integral technique is used to study the ultraviolet or short distance properties of the Kardar-Parisi-Zhang (KPZ) equation with white Gaussian noise. We apply this technique to calculate the one-loop effective potential for the $\mathrm{KPZ}$ equation. The effective potential is (at least) one-loop ultraviolet renormalizable in 1,2 , and 3 space dimensions, but non-renormalizable in 4 or higher space dimensions. This potential is intimately related to the probability distribution function $(\mathrm{PDF})$ for the spacetime averaged field. For the restricted class of field configurations considered here, the KPZ equation exhibits dynamical symmetry breaking (DSB) via an analog of the Coleman-Weinberg mechanism in 1 and 2 space dimensions, but not in 3 space dimensions.

PACS: 02.50.Ey; 02.50.-r; 05.40.+j; cond-mat/9904413

Keywords: effective potential, KPZ equation, dynamical symmetry breaking

In understanding the onset of spatio-temporal pattern formation in systems out of equilibrium, it has proven extremely useful to begin the pattern formation and selection analysis by first obtaining and then classifying all the static and spatially homogeneous states allowed by the time dependent partial differential equations that model the system in question [1]. In this way, one can decide whether the system will exhibit Hopf bifurcations and/or Turing instabilities and get a handle on the qualitative nature of the patterns expected to emerge. This can be followed up by an amplitude analysis of the fluctuations about these static and homogeneous configurations. The unstable modes are the ones that lead to nontrivial patterns. For out-of-equilibrium systems coupled to noisy environments (or with inherent internal noise) it is important to know how the stochastic sources can alter and shift these static and homogeneous configurations, since these affect the onset of the pattern-forming instabilities. Here we show how the effects of noise on these configurations can be computed using functional integral methods, and reveal an intrinsically ultraviolet one-loop phenomenon not captured by the more common methods of analysis described above: namely the occurrence of dimension dependent dynamical symmetry breaking. In this paper we focus attention on the Kardar-ParisiZhang (KPZ) equation [2,3]

$$
\left(\frac{\partial}{\partial t}-\nu \vec{\nabla}^{2}\right) \phi=F_{0}+\frac{\lambda}{2}(\vec{\nabla} \phi)^{2}+\eta .
$$

The KPZ equation arises in a host of seemingly distinct physical contexts and problems ranging from models of turbulence [4,5], interface growth, driven diffusion and flame fronts 3, directed polymers in a random medium [6,7], certain lattice gases with hard-core exclusion [8], and even structure development in the early universe [9]. In the fluid dynamics interpretation (when the KPZ equation is used as a model of Burgers turbulence), the fluid velocity is $\vec{v}=-\vec{\nabla} \phi$ and the KPZ field plays the rôle of a velocity potential. In the surface growth interpretation $\phi(\vec{x}, t)$ is the local height of the surface, defined over a two-dimensional plane [2]. The constant "tadpole" term $F_{0}$ in (11) is necessary for the ultraviolet regularization 10 of the KPZ equation After renormalization, we will argue that the tadpole can and should be set to zero.

If the noise $\eta$ is Gaussian, all stochastic averages are encoded in the generating functional] [10]

$$
\begin{aligned}
& Z[J]=\int(\mathcal{D} \phi) \exp \left(\int J \phi\right) \\
& \exp \left(-\frac{1}{2} \iint\left[\partial_{t} \phi-\nu \vec{\nabla}^{2} \phi-F_{0}-\frac{\lambda}{2}(\vec{\nabla} \phi)^{2}\right]\right. \\
&\left.G_{\eta}^{-1}\left[\partial_{t} \phi-\nu \vec{\nabla}^{2} \phi-F_{0}-\frac{\lambda}{2}(\vec{\nabla} \phi)^{2}\right]\right)
\end{aligned}
$$

In general, a functional Jacobian determinant must be included. For the KPZ equation this determinant is a field-independent constant 10. For translation-invariant Gaussian noise, we split its two-point function into an amplitude, $\mathcal{A}$, and a shape, $g_{2}(x, y)$ 10

$$
G_{\eta}(x, y) \stackrel{\text { def }}{=} \mathcal{A} g_{2}(x-y)
$$

with the convention that

\footnotetext{
${ }^{1}$ In this regard it is interesting to point out that small scale properties of a randomly stirred fluid were studied some years ago by Yakhot, who found that the ultraviolet renormalizability of the forced Navier-Stokes (NS) equation required the addition of a term not originally present in the bare NS equation [11].

2This "direct" functional integral formalism is different (yet equivalent) from the more extended MSR approach 18.
} 


$$
\int g_{2}^{-1}(\vec{x}, t) \mathrm{d}^{d} \vec{x} \mathrm{~d} t=1=\tilde{g}_{2}^{-1}(\vec{k}=\overrightarrow{0}, \omega=0) .
$$

The amplitude $\mathcal{A}$ is the loop-counting parameter for this theory [10].

There are two important symmetries of the KPZ equation that are relevant for our analysis. First, we have

$$
\begin{aligned}
\phi & \rightarrow \phi+c(t), \\
F_{0} & \rightarrow F_{0}+\frac{d c(t)}{d t} .
\end{aligned}
$$

In the fluid dynamics interpretation this symmetry is a "gauge transformation" of the scalar field $\phi$ that does not change the fluid velocity $\vec{v}$. In the surface growth interpretation this symmetry is a (Type I) Galilean transformation. It can be used to eliminate any spurious motion of the background field. The second symmetry is $(|\epsilon \ll 1|)$

$$
\begin{aligned}
\vec{x} & \rightarrow \vec{x}^{\prime}=\vec{x}-\lambda \vec{\epsilon} t, \\
t & \rightarrow t^{\prime}=t, \\
\phi(\vec{x}, t) & \rightarrow \phi^{\prime}\left(\vec{x}^{\prime}, t^{\prime}\right)=\phi(\vec{x}, t)-\vec{\epsilon} \cdot \vec{x} .
\end{aligned}
$$

In the fluid dynamics interpretation this symmetry is equivalent to a (Type II) Galilean transformation of the fluid velocities

$$
\vec{v} \rightarrow \vec{v}^{\prime}=\vec{v}+\vec{\epsilon} .
$$

In the surface growth interpretation this symmetry amounts to tilting the coordinate system at an angle to the vertical, with

$$
\tan (\theta)=\|\vec{\epsilon}\| .
$$

This Type II Galilean transformation is an exact invariance of the zero-noise KPZ equation, but once noise is added to the system, it will remain a symmetry only if the noise is translation-invariant and temporally white.

A few words are in order regarding the choice of field configurations used for calculating the effective potential. In quantum field theory (QFT) patterns of spontaneous and dynamical symmetry breaking are revealed using static and homogeneous fields, since these configurations minimize the positive kinetic energy contribution to the total energy. The QFT ground states (basic field configurations) are therefore determined by searching for the minima of the effective potential. It turns out that an effective potential for the KPZ equation can be defined for a static, but spatially inhomogeneous field configuration, if it has the form

$$
\phi=-\vec{v} \cdot \vec{x} .
$$

In the hydrodynamic interpretation this corresponds to a stationary and homogeneous fluid flow [5] with velocity $\vec{v}$. In the surface growth interpretation $\|\vec{v}\|$ corresponds to a constant slope of the surface [2] (linear ramp). Due to the structure of the stochastic field theory (2) leading to the KPZ equation, this is the most general static configuration allowing one to define an effective potential. This is because the effective potential must be a function of a static field that satisfies the heat equation: $\partial_{t} \phi-\nu \nabla^{2} \phi=0$. Any other field configuration more general than (12) (and/or time dependent) would force us to deal with the complexities of the effective action.

Following the general construction in [10], the KPZ zero-loop effective potential is given by

$$
\mathcal{V}_{\text {zero-loop }}[v]=\frac{1}{2}\left[\left(F_{0}+\frac{1}{2} \lambda v^{2}\right)^{2}-F_{0}^{2}\right] .
$$

The effective potential is generally calculated in terms of a reference background field $\phi_{0}=-\vec{v}_{0} \cdot \vec{x}$. We have used the Type II symmetry to set $\vec{v}_{0}=\overrightarrow{0}$. This potential is formally equivalent to that of $\lambda \phi^{4}$ QFT - with the velocity $\vec{v}$ playing the role of the quantum field $\phi_{\mathrm{QFT}}$. Even at zero-loops we see that for $F_{0}<0$, the effective potential takes on the "Mexican hat" form, so that the onset of spontaneous symmetry breaking (SSB) would not be at all unexpected 14,15. However, because of the Type I symmetry, the renormalized value of $F_{0}$ must be set to zero, and SSB is not encountered in the KPZ equation. (There is an instructive analogy with the renormalization program for massless $\lambda \phi^{4}$ QFT. It is well known that for this QFT one still needs to introduce a bare mass parameter that must be kept for all intermediate stages of the regularization. It is only after renormalization is complete that it makes sense to set the renormalized value of the mass to zero.) Considerably more subtle is the onset of DSB which can be detected only via a one-loop computation.

Evaluating the general expression for the one-loop effective potential computed in [10] for the case of the KPZ equation, we obtain

$$
\begin{aligned}
\mathcal{V}[v] & =\frac{1}{2}\left(F_{0}+\frac{1}{2} \lambda v^{2}\right)^{2} \\
& +\frac{1}{2} \mathcal{A} \int \frac{\mathrm{d}^{d} \vec{k} \mathrm{~d} \omega}{(2 \pi)^{d+1}} \log \left[1+\frac{\tilde{g}_{2}(\vec{k}, \omega) \lambda\left(F_{0}+\frac{1}{2} \lambda v^{2}\right) \vec{k}^{2}}{(\omega-\lambda \vec{v} \cdot \vec{k})^{2}+\nu^{2}\left(\vec{k}^{2}\right)^{2}}\right] \\
& -(\vec{v} \rightarrow \overrightarrow{0})+O\left(\mathcal{A}^{2}\right) .
\end{aligned}
$$

Because we have chosen noise which is white in time, so that $\tilde{g}_{2}(\vec{k}, \omega) \rightarrow \tilde{g}_{2}(\vec{k})$, we can shift the integration variable from $\omega$ to $\omega-\lambda \vec{v} \cdot \vec{k}$. The resulting integral becomes

$$
\begin{aligned}
\mathcal{V}[v] & =\frac{1}{2}\left[\left(F_{0}+\frac{1}{2} \lambda v^{2}\right)^{2}-F_{0}^{2}\right]+\frac{1}{2} \mathcal{A} \int \frac{\mathrm{d}^{d} \vec{k} \mathrm{~d} \omega}{(2 \pi)^{d+1}} \\
& \times \log \left[\frac{\omega^{2}+\nu^{2}\left(\vec{k}^{2}\right)^{2}+\tilde{g}_{2}(\vec{k}) \lambda\left(F_{0}+\frac{1}{2} \lambda v^{2}\right) \vec{k}^{2}}{\omega^{2}+\nu^{2}\left(\vec{k}^{2}\right)^{2}+\tilde{g}_{2}(\vec{k}) \lambda F_{0} \vec{k}^{2}}\right] \\
& +O\left(\mathcal{A}^{2}\right) .
\end{aligned}
$$

We point out that the denominator of the logarithm in the integrand is independent of the velocity field $\vec{v}$. This 
important result is a consequence of the fact that the Jacobian functional determinant encountered in 10 is constant for the KPZ equation. One can perform the frequency integral exactly to obtain

$$
\begin{aligned}
\mathcal{V}[v] & =\frac{1}{2}\left[\left(F_{0}+\frac{1}{2} \lambda v^{2}\right)^{2}-F_{0}^{2}\right] \\
& +\frac{1}{2} \mathcal{A} \int \frac{\mathrm{d}^{d} \vec{k}}{(2 \pi)^{d}}\left\{\sqrt{\left[\nu^{2}\left(\vec{k}^{2}\right)^{2}+\tilde{g}_{2}(\vec{k}) \lambda\left(F_{0}+\frac{1}{2} \lambda v^{2}\right) \vec{k}^{2}\right]}\right. \\
& \left.-\sqrt{\left[\nu^{2}\left(\vec{k}^{2}\right)^{2}+\tilde{g}_{2}(\vec{k}) \lambda F_{0} \vec{k}^{2}\right]}\right\}+O\left(\mathcal{A}^{2}\right) .
\end{aligned}
$$

The bare KPZ potential (13) contains terms proportional to $v^{0}, v^{2}$, and $v^{4}$. (The one-loop contribution (15), expanded in powers of $v^{2}$, has terms proportional to $v^{2 n}$.) Let us now take the spatial noise spectrum to be cutoff white noise, i.e.,

$$
\tilde{g}_{2}(\vec{k})=\tilde{g}_{2}(\|\vec{k}\|)=\Theta(\Lambda-k) .
$$

With this choice of noise, the $v^{2}$ term is proportional to $\Lambda^{d}$, the $v^{4}$ term to $\Lambda^{d-2}$, and the $v^{6}$ term to $\Lambda^{d-4}$. In order to be able to absorb the infinities into the bare action we must have $d<4$. Note that $d=4$ is a marginal case, and one might suspect that $d=4$ is a critical dimension for the KPZ equation [6]7]. That is: the KPZ equation (subject to white noise) is one-loop ultraviolet renormalizable only in 1, 2, and 3 spatial dimensions. Even so, one-loop renormalizability requires an explicit tadpole. (Without a tadpole there is no term proportional to $v^{2}$ in the zero-loop potential, and hence no possibility of regularizing the leading divergence.) Strictly speaking, the claim of one-loop renormalizability requires investigation of the effective action (wave-function renormalization). A one-loop ultraviolet renormalization of the effective $a c$ tion, valid for time dependent and inhomogeneous fields, has been carried out which fully supports the effective potential calculations presented here [13].

The ultraviolet renormalizability of the KPZ equation depends critically on the ultraviolet behavior of the noise. Let us suppose that the noise is power-law distributed in the ultraviolet region with $g_{2}(k) \approx\left(k_{0} / k\right)^{\theta} \Theta(\Lambda-k)$. In this case the $n$th term in the expansion has ultraviolet behavior proportional to $v^{2 n} \Lambda^{d+2-2 n-n \theta}$. The KPZ equation is then one-loop ultraviolet renormalizable for $d<4+3 \theta$. Thus, the one-loop ultraviolet renormalizability can be extended to dimensions four and greater depending on how the noise scales in the ultraviolet region. We will not pursue these issues further in this paper and henceforth restrict our attention to white noise.

There is a formal connection with $\lambda\left(\phi^{4}\right)_{[d+1]+1}$ QFT that is made apparent by extracting a factor of $\nu^{2} \vec{k}^{2}$ from equation (15). If we do so, we obtain

$$
\begin{aligned}
\mathcal{V}[v]= & \frac{1}{2}\left[\left(F_{0}+\frac{1}{2} \lambda v^{2}\right)^{2}-F_{0}^{2}\right] \\
& +\frac{1}{2} \mathcal{A} \nu \int_{0}^{\Lambda} \frac{\mathrm{d}^{d} \vec{k}|k|}{(2 \pi)^{d}}\left\{\sqrt{\vec{k}^{2}+\frac{\lambda}{\nu^{2}}\left(F_{0}+\frac{1}{2} \lambda v^{2}\right)}\right.
\end{aligned}
$$

$$
\left.-\sqrt{\vec{k}^{2}+\frac{\lambda}{\nu^{2}} F_{0}}\right\}+O\left(\mathcal{A}^{2}\right) .
$$

This is recognizable as the effective potential for $\lambda \phi^{4}$ QFT in $d+1$ spatial dimensions, that is, Euclidean $[d+1]+1$ spacetime dimensions 14, 15. To make the connection, interpret $\lambda F_{0} / \nu^{2}$ as the mass term $m^{2}$ of the QFT, $\lambda^{2} / \nu^{2}$ as $\lambda_{\mathrm{QFT}}$ (the coupling constant of the QFT), and $v$ as the field $\phi_{\mathrm{QFT}}$.

After renormalization the tadpole is to be set to zero, but we still need a bare tadpole to act as a counterterm during the intermediate stages of the regularization. This is completely analogous to the situation in massless $\phi^{4}$ QFT, where the renormalized mass of the quantum field is fine tuned to zero by hand [14,15.

We now evaluate the effective potential in different spatial dimensions.

$\underline{\text { Case } d=1:}$ We are interested in the integral

$$
\mathcal{I}(a) \stackrel{\text { def }}{=} \int_{0}^{+\infty} \mathrm{d} k^{2}\left[\sqrt{k^{2}+a}-\sqrt{k^{2}}\right] .
$$

This integral is divergent and needs to be regularized. The most direct way to do so is by the "differentiate and integrate" trick which leads to [14]

$$
\mathcal{I}(a)=\kappa a-\frac{2}{3} a^{3 / 2} .
$$

Here $\kappa$ is an infinite constant of integration. We absorb $\kappa$ into the bare action, where it renormalizes $F_{0}$ 14 15

$$
\begin{aligned}
\mathcal{V}[v ; d=1]= & \frac{1}{2}\left[\left(F_{0}+\frac{1}{2} \lambda v^{2}\right)^{2}-F_{0}^{2}\right] \\
& -\frac{1}{6 \pi} \mathcal{A} \frac{\lambda^{3 / 2}}{\nu^{2}}\left[\left(F_{0}+\frac{1}{2} \lambda v^{2}\right)^{3 / 2}-F_{0}^{3 / 2}\right] \\
& +O\left(\mathcal{A}^{2}\right) .
\end{aligned}
$$

These are all renormalized parameters at $O(\mathcal{A})$. The zero-loop contribution will always dominate at large fields (rapid flow). Near $v=0$, it is the one-loop contribution that is dominant. We take the physical value of the renormalized tadpole, $F_{0}=0$, and encounter something very interesting - the system undergoes dynamical symmetry breaking (DSB) in a manner qualitatively similar to the Coleman-Weinberg mechanism of QFT. The effective potential then simplifies to

$$
\mathcal{V}[v ; d=1]=\frac{\lambda^{2}}{8} v^{4}-\frac{1}{6 \pi} \mathcal{A} \frac{\lambda^{3}}{2^{3 / 2} \nu^{2}}|v|^{3}+O\left(\mathcal{A}^{2}\right) .
$$

The potential is not analytic at zero field (a phenomenon well known from massless QFTs) [14,15. For large $v$ the classical potential dominates, whereas for small $v$ oneloop effects dominate. Thus the symmetric vacuum $(v=$ 0 ) is unstable. While it is easy to see that the symmetry is dynamically broken, the presence of the unknown $O\left(\mathcal{A}^{2}\right)$ terms make it difficult to make a quantitative estimate for $v_{\min }$. We can nevertheless qualitatively estimate the shift in the expectation value of the velocity field 


$$
v_{\text {min }}= \pm \mathcal{A} \frac{\lambda}{2 \pi 2^{1 / 2} \nu^{2}}+O\left(\mathcal{A}^{2}\right) .
$$

This DSB is particularly intriguing in that it suggests the possibility of a noise driven pump. For example, in thin pipes (where the flow is essentially one-dimensional) and provided the physical situation justifies the use of Burgers equation, this result indicates the presence of a bimodal instability leading to the onset of a fluid flow with velocity depending on noise amplitude.

Case $d=2$ : Because of the analogy between the oneloop effective potential for the KPZ equation (for white noise) and that for the $\lambda \phi^{4}$ QFT, we can write down the renormalized one-loop effective potential by inspection 14, 15,

$$
\begin{aligned}
& \mathcal{V}[v ; d=2]=\frac{1}{2}\left\{\left[F_{0}(\mu)+\frac{1}{2} \lambda(\mu) v^{2}\right]^{2}-\left[F_{0}(\mu)\right]^{2}\right\} \\
& \quad+\frac{1}{2} \mathcal{A} \frac{1}{(2 \pi)^{2}} \frac{\lambda^{2}}{\nu^{3}}\left\{\left[F_{0}(\mu)+\frac{1}{2} \lambda(\mu) v^{2}\right]^{2}\right. \\
& \left.\quad \times \log \left[\frac{F_{0}(\mu)+\frac{1}{2} \lambda(\mu) v^{2}}{\mu^{2}}\right]-\left[F_{0}(\mu)\right]^{2} \log \left[\frac{F_{0}(\mu)}{\mu^{2}}\right]\right\} \\
& \quad+O\left(\mathcal{A}^{2}\right) .
\end{aligned}
$$

Here $\mu$ is the renormalization scale in the sense it is used in QFT [14,15.

We now tune the renormalized tadpole to its physical value of zero. After performing a finite renormalization to simplify the expression, we obtain

$$
\mathcal{V}[v ; d=2]=\frac{\lambda^{2}}{8} v^{4}+\mathcal{A} \frac{1}{(2 \pi)^{2}} \frac{\lambda^{4}}{8 \nu^{3}} v^{4} \log \left(\frac{v^{2}}{\mu^{2}}\right)+O\left(\mathcal{A}^{2}\right) .
$$

The one-loop contribution will always dominate at large fields (rapid flow). Near $v=0$ the logarithmic singularity is rendered finite by the polynomial prefactor. That the KPZ potential has a non-trivial minimum exhibiting DSB is exactly the analog of the Coleman-Weinberg mechanism 14,15. We can estimate the location of the minimum by differentiating the effective potential. We obtain

$$
v_{\min }= \pm \mu \exp \left[-\frac{(2 \pi)^{2} \nu^{3}}{2 \lambda^{2} \mathcal{A}}-\frac{1}{4}+O(\mathcal{A})\right] .
$$

Note that $v_{\min } \rightarrow 0$ as $\mathcal{A} \rightarrow 0$, to recover the tree level minimum $v_{\text {min }}=0$. The present discussion is relevant to either (1) surface evolution on a two dimensional substrate, or (2) thin superfluid films, since superfluids are automatically vorticity free, justifying the application of the KPZ equation.

We can also calculate the one-loop beta function, for as the bare effective potential does not depend on the renormalization scale, one has that $\mu \mathrm{d} \mathcal{V} / \mathrm{d} \mu=0$. We get

$$
\beta_{\lambda} \stackrel{\text { def }}{=} \mu \frac{\mathrm{d}}{\mathrm{d} \mu} \lambda=\frac{\mathcal{A}}{4 \pi^{2}} \frac{\lambda^{2}}{\nu^{3}} \lambda+O(\mathcal{A})^{2} .
$$

$\underline{\text { Case } d=3:}$ We are interested in the integral

$$
\mathcal{I}\left(a^{2}\right) \stackrel{\text { def }}{=} \int_{0}^{+\infty} \mathrm{d} k^{2} k^{2}\left[\sqrt{k^{2}+a^{2}}-\sqrt{k^{2}}\right] .
$$

The "differentiate and integrate" trick yields 14

$$
\mathcal{I}\left(a^{2}\right)=\kappa_{1} a^{2}+\kappa_{2} a^{4}+\frac{4}{15} a^{5} .
$$

Here $\kappa_{1}$ and $\kappa_{2}$ are two infinite constants of integration. Since the sign in front of the $a^{5}$ term is positive there is no possibility of DSB in $d=3$. We absorb $\kappa_{1}$ and $\kappa_{2}$ into the bare action, where they renormalize both $F_{0}$ and $\lambda$

$$
\begin{aligned}
\mathcal{V}[v ; d=3]= & \frac{1}{2}\left[\left(F_{0}+\frac{1}{2} \lambda v^{2}\right)^{2}-F_{0}^{2}\right] \\
& +\frac{1}{\left(30 \pi^{2}\right)} \mathcal{A} \frac{\lambda^{5 / 2}}{\nu^{4}}\left[\left(F_{0}+\frac{1}{2} \lambda v^{2}\right)^{5 / 2}-F_{0}^{5 / 2}\right] \\
& +O\left(\mathcal{A}^{2}\right) .
\end{aligned}
$$

These are all renormalized parameters at order $O(\mathcal{A})$. For large fields the one-loop correction is dominant, whereas for small fields the tree level result dominates. The effective potential is positive and monotonically increasing.

If we set the renormalized tadpole to its physical value of zero, we have

$$
\mathcal{V}[v ; d=3]=\frac{\lambda^{2}}{8} v^{4}+\frac{1}{\left(30 \pi^{2}\right)} \mathcal{A} \frac{\lambda^{5}}{2^{5 / 2} \nu^{4}}|v|^{5}+O\left(\mathcal{A}^{2}\right) .
$$

At zero-loops the vacuum is symmetric at $v_{\min }=0$. Oneloop physics does not change this. There is an important sign change in the one-loop contribution when comparing $d=3$ with $d=1$. The DSB that is so interesting in $d=1$ and $d=2$, is now absent in $d=3$. Notice that the effective potential is non-analytic at $v_{\min }=0$. This case is of most interest in hydrodynamics and we have shown that (at one-loop) the ground state $\left(v_{\min }=0\right)$ is stable under Gaussian noise.

Discussion: When taken as a model for turbulence, it is natural to study the ultraviolet properties of the KPZ equation because in a turbulent fluid, energy cascades down from larger to smaller length scales and one might expect scale-invariant solutions in the limit as $k \rightarrow+\infty$. Indeed, Forster, Nelson, and Stephen pointed out long ago the importance of studying the short distance and short time (i.e., ultraviolet) correlations in the fluid velocity in fully developed turbulence [4]. More recently, Yakhot [11] undertook an analysis of the ultraviolet properties of the randomly forced Navier-Stokes (NS) equation and found that ultraviolet renormalizability requires modifying the fluid dynamical equation by a term not originally present in the NS equation, though this new term is much more complicated than the tadpole needed to successfully renormalize the KPZ equation.

The ultraviolet properties of the KPZ equation exposed in this paper reveal a rich structure and complements studies concerned with the long distance (infrared) 
features of this equation. This distinction shows up in a striking way in both, the need of a bare tadpole for ultraviolet renormalizability and the presence of dimension dependent dynamical symmetry breaking for the configurations studied here. Our focus in this paper has been on the concept of the effective potential and we have shown how this concept, so useful in QFT, can be extended to stochastic equations containing gradient forcing terms, such as the KPZ equation, by employing inhomogeneous and static fields.

The evidence for the dynamical symmetry breaking found here must be interpreted with due caution. We must emphasize, for the reasons explained earlier, that the effective potential is calculated for a restricted class of field configurations and the symmetry breaking is with respect to this class of constant fluid velocity configurations. Thus, while for $d=3$ the one-loop effective potential is minimized for a zero-velocity background flow $v_{\min }=0$, the potential in both $d=1$ and $d=2$ is minimized at a non-zero value of the fluid flow, namely (22) and (25), respectively. The symmetry breaking is thus manifested as a preference for the system to "jump" from zero to a non-zero but constant flow. The jump is triggered, of course, by the noise source. In the surface growth interpretation, the minimum of the potential for $d=3$ corresponds to a surface with zero slope, while the dynamical symmetry breaking that occurs in $d=2$ and $d=1$ corresponds to a jump in the surface slope to a constant non-zero value. This should be contrasted to recent exact and numerical calculations of the $\mathrm{PDF}$ for the KPZ equation in $d=1$ dimension. By making use of a lattice version of the KPZ equation in one dimension, Derrida and Lebowitz have performed an exact calculation of the probability distribution of the deviation of the average current [8]. The results of these authors agree with a numerical calculation of the KPZ PDF in $d=1$ by Yakhot and Chekhlov [12]. In these papers, the PDF, which is essentially the exponential of the effective potential, gives information about the probability to find a velocity difference $\Delta u_{r}=v(x+r)-v(x)$ at two widely separated points in the fluid (i.e., as $r \rightarrow+\infty$ ) and for asymptotically large times. Their PDFs have a global maximum at $\Delta u_{r}=0$, corresponding to no velocity difference, i.e., a spatially constant velocity (or a constant slope surface). Thus, the most probable configuration corresponds to a spatially constant velocity. Of course, these PDFs take into account all dynamically allowed configurations that evolve via the KPZ equation; our PDF, by contrast, samples only the class of constant velocity configurations. Nevertheless, and within these limitations, we have been able to show that for the linear theory $(\lambda=0)$ the ground state has zero velocity (or zero slope) and that once we turn on the nonlinear interaction, and restrict the fluid to have a constant velocity, there is a dynamically preferred velocity as determined by one-loop physics in $d=1$ and $d=2$ dimensions.

In this paper we have also demonstrated that the KPZ equation with white noise is at least one-loop renormaliz- able for $d=1,2,3$ dimensions but is non-renormalizable (at one-loop) for $d>4$. In making statements about renormalizability, care must be taken in distinguishing ultraviolet and infrared properties. Thus, from the work of Sun and Plischke [16] and Frey and Täuber [17], it is known that the $\mathrm{KPZ}$ equation is, at least, two-loop renormalizable for $d=1,2,3$. Although these authors were interested in the infrared properties of the KPZ equation, they had to deal with both ultraviolet and infrared divergences in their work. By making use of the mapping of the KPZ equation to directed polymers with quenched noise, the one-loop renormalizability has been demonstrated in $2<d<4$ [7].

Though the calculations presented here are limited to one-loop order in the noise amplitude, it must be emphasized that in many cases one-loop physics is enough to capture essential features of stochastic partial differential equations (SPDEs) [10] and QFTs. Furthermore, while calculations of the one-loop effective potential are straightforward, one should not forget that it is necessary to develop a physical interpretation of this effective potential to ensure that it is as relevant for SPDEs, as it is for QFTs 10. In this paper we have presented an analogy between the statics of the KPZ equation and the static behavior of $\lambda \phi^{4} \mathrm{QFT}$ - the vacuum structure of the $\phi^{4}$ QFT carries over into the basic field configurations of the KPZ equation. In 1 and 2 space dimensions we have exhibited the occurrence of DSB, which does not take place in 3 space dimensions.

\footnotetext{
$+\quad$ Electronic mail: hochberg@laeff.esa.es

+ Electronic mail: carmen@t6-serv.lanl.gov

+++ Electronic mail: mercader@laeff.esa.es

++++ Electronic mail: visser@kiwi.wustl.edu

[1] M.C. Cross and P.C. Hohenberg, Rev. Mod. Phys. 65, 851 (1993).
}

[2] M. Kardar, G. Parisi, and Y-C. Zhang, Phys. Rev. Lett. 56, 889 (1986).

[3] E. Medina, T. Hwa, M. Kardar, and Y-C. Zhang, Phys. Rev. A 39, 3053 (1989).

[4] D. Forster, D.R. Nelson and M.J. Stephen, Phys. Rev. A 16, 732 (1977).

[5] U. Frisch, Turbulence (Cambridge University Press, Cambridge, England, 1995).

[6] M. Lässig and H. Kinzelbach, Phys. Rev. Lett. 78, 903 (1997).

[7] R. Bundschuh and M. Lässig, Phys. Rev. E 54, 304 (1996).

[8] B. Derrida and J. Lebowitz, Phys. Rev. Lett. 80, 209 (1998).

[9] A. Berera and L-Z. Fang, Phys. Rev. Lett. 72, 458 (1994); D. Hochberg and J. Pérez-Mercader, Gen. Rel. Gravit. 28, 1427 (1996); T. Goldman, D. Hochberg, R. Laflamme, and J. Pérez-Mercader, Phys. Lett. A 
222, 177 (1996); J.F. Barbero, A. Domínguez, T. Goldman, and J. Pérez-Mercader, Europhys. Lett. 38, 637 (1997); A. Domínguez, D. Hochberg, J.M. MartínGarcía, J. Pérez-Mercader, and L.S. Schulman, Astron. and Astrophys. 344, 27 (1999).

[10] D. Hochberg, C. Molina-París, J. Pérez-Mercader, and M. Visser, Phys. Rev. E 60, 6343 (1999).

[11] V. Yakhot, Phys. Rev. A 23, 1486 (1981).

[12] V. Yakhot and A. Chekhlov, Phys. Rev. Lett. 77, 3118 (1996).

[13] D. Hochberg, C. Molina-París, J. Pérez-Mercader and M. Visser, Seeley-DeWitt coefficients, heat kernel renormalization and effective action for the KardarParisi-Zhang equation, in preparation.

[14] S. Weinberg, The quantum theory of fields I \& II (Cambridge University Press, England, 1996).

[15] J. Zinn-Justin, Quantum field theory and critical phenomena (Oxford University Press, England, 1996).

[16] T. Sun and M. Plischke, Phys. Rev. E 49, 5046 (1994).

[17] E. Frey and U.C. Täuber, Phys. Rev. E 50, 1024 (1994).

[18] P.C. Martin, E.D. Siggia, and H.A. Rose, Phys. Rev. A 8, 423 (1973). 